\title{
Synthesis of Copper Oxides Nanostructures by an Overstressed Nanosecond Discharge in Atmospheric Pressure Air between Copper Electrodes
}

\author{
Alexander K. Shuaibov, Alexander Y. Minya, Antonina A. Malinina, Alexander N. Malinin, \\ Vladislav V. Danilo, Mihail Yu. Sichka, Igor V. Shevera \\ Department of Quantum Electronics, Faculty of Physics, Uzhhorod National University, Uzhgorod, Ukraine
}

Email address:

alexsander.shuaibov@uzhnu.edu.ua (A. K. Shuaibov)

\section{To cite this article:}

Alexander K. Shuaibov, Alexander Y. Minya, Antonina A. Malinina, Alexander N. Malinin, Vladislav V. Danilo, Mihail Yu. Sichka, Igor V. Shevera. Synthesis of Copper Oxides Nanostructures by an Overstressed Nanosecond Discharge in Atmospheric Pressure Air between Copper Electrodes. American Journal of Mechanical and Materials Engineering. Vol. 2, No. 1, 2018, pp. 8-14.

doi: $10.11648 /$ j.ajmme.20180201.12

Received: February 13, 2018; Accepted: April 8, 2018; Published: May 7, 2018

\begin{abstract}
The results of study of the characteristics and parameters of plasma of a high-current nanosecond discharge in atmospheric pressure air between copper electrodes under conditions of strong overstress of the discharge gap are presented. Under such conditions, favorable conditions are created for igniting an atmospheric pressure diffuse discharge in an inhomogeneous electric field. The spatial, electrical and optical characteristics of the discharge, as well as the parameters of the air plasma with small additions of copper vapor are studied. It is shown that this type of discharge can be used for the synthesis of copper oxides nanostructures.
\end{abstract}

Keywords: Copper Oxides, Nanostructures, Diffuse Discharge

\section{Introduction}

Nanostructures of transition metal oxides are now widely used in various technical devices and technologies, medicine, in particular, and in modern solar cells [1-3]. In addition to various modifications of chemical methods for the synthesis of copper and zinc nanostructures, as well as their oxides, physical methods for the synthesis of similar nanostructures that also develop intensively are promising. The main directions of synthesis of such nanostructures are the use of laser plasma in vacuum or liquid conditions [4, 5]. A promising method for synthesizing nanostructures of metals and their oxides is a pulsed discharge in distilled water or ethyl alcohol between metal electrodes [6, 7]. But these methods of synthesis have a number of drawbacks-the high cost of laser and vacuum equipment and the low yield of useful materials. For powerful nanosecond discharges in a liquid, the rapid burnout of electrodes is characteristic, which leads to a change in the conditions for the synthesis of nanostructures. Therefore, it is actual to search for other electric-discharge technologies for the synthesis of nanostructures that would be based on a high-voltage diffuse discharge of nanosecond duration in atmospheric pressure air without the use of special preionization systems. In such discharges, which are ignited in a highly nonuniform electric field, the role of the preionization system is performed by ultraviolet or X-ray radiation, as well as a beam of runaway electrons [8, 9]. Thus, in work [10] it was reported on the successful application of a diffuse discharge with runaway electrodes for purification and oxidation of the aluminum surface. Promising for the synthesis of colloidal solutions of nanostructures of transition metal oxides is also a high-current nanosecond discharge between a metallic and liquid nonmetallic electrode based on solutions of metal salts in atmospheric pressure air [11, 12].

In this paper, we present the results of an investigation of the synthesis conditions and some optical characteristics of thin nanostructured films based on copper and dissociation products of air molecules in an overstressed nanosecond discharge.

\section{Technique and Experimental Conditions}

The design of the discharge chamber with a system of 
electrodes between which a nanosecond discharge in air was ignited is shown in Figure 1. Electrodes made of copper were installed in a chamber of dielectric with a volume 3 liters. Most of the experiments were carried out at atmospheric air pressure, but the discharge chamber could be evacuated to a residual pressure $10 \mathrm{~Pa}$. The excess air pressure in the chamber could reach 3 Atm.

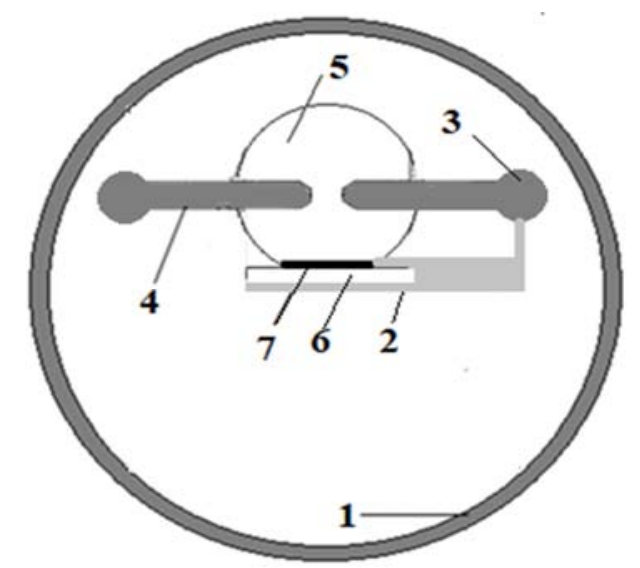

Figure 1. Diagram of a gas-discharge cell: 1 - housing of a dielectric cell, 2 - system for mounting a substrate for deposition a thin film, 3 - unit for regulating the interelectrode distance, 4 - discharge electrodes, 5 - area of space where deposition of electrode material can occur, 6 - glass substrate for thin films deposition, 7 - a film from a sputtered electrode material.

To reduce the effect of electromagnetic interference on the system for recording the characteristics of a high-current nanosecond discharge, the chamber with the electrode system was mounted in a screen made of a metal grid. The diameter of the cylindrical copper electrodes was $5 \mathrm{~mm}$, and the radius of the rounding of the working end part of the electrodes was $3 \mathrm{~mm}$. To locate the discharge in the interelectrode gap, the electrodes were inserted into dielectric tubes made of fluoroplastic, so that the length of their non-isolated part did not exceed 3-4 mm.

To ignite the discharge, high voltage bipolar pulses of a total duration 50-100 ns and an amplitude $\pm(20-40) \mathrm{kV}$ were applied to the electrodes. In this case, a diffuse discharge with an amplitude of current pulses 50-170 A was ignited between the tips of the electrodes. The plasma volume did not exceed 5-10 $\mathrm{mm}^{3}$. With an interelectrode distance 1.0 $\mathrm{mm}$, the discharge gap was strongly overvstressed. Nanosecond discharge at air pressure $p=1-3$ Atm was quite homogeneous. The repetition rate of pulses varied in the range $\mathrm{f}=40-1000 \mathrm{~Hz}$. The distance between the copper electrodes was $1 \mathrm{~mm}$.

The voltage pulses at the discharge gap and the discharge current were measured using a wide-band capacitor divider, Rogowski coil, and the wide-wave oscilloscope 6-LOR-04. The temporal resolution of this recording system was 2-3 ns. The study of the spatial characteristics of the discharge was carried out using a digital camera. To record plasma emission spectra, we used MDR-2 monochromator, a photomultiplier FEU-106, a direct current amplifier, and an electronic potentiometer. Radiation of the discharge plasma was analyzed in the spectral range 200-650 nm. The plasma emission detection system was calibrated by the radiation of a deuterium lamp in the spectral range $200-400 \mathrm{~nm}$ and ganglamps in the 400-650 $\mathrm{nm}$ range.

The transmission spectra of radiation by films that were synthesized on glass substrates were recorded with the OCEAN OPTICS USB 2000 spectrometer. Probe radiation was fed to the substrate with the film using an optical fiber system. The films were deposited for 20-40 minutes when the glass substrate was placed $2-3 \mathrm{~cm}$ from the center of the discharge. The analysis of the film surface was carried out using a scanning electron microscope.

The photoluminescence spectra of thin films were studied by irradiating a film with a high-pressure mercury arc lamp at three wavelengths $(279,330,380 \mathrm{~nm})$. Radiation of a mercury arc lamp in the spectral range $5 \mathrm{~nm}$ was allocated at centers with wavelengths $279,330,380 \mathrm{~nm}$ with a help of a monochromator and directed to the film. The photoluminescence spectrum of nanostructures was recorded with the aid of another monochromator equipped with a photoelectric radiation detection system.

\section{Results and Discussion}

\subsection{Characteristics and Parameters of the Discharge Plasma in Air between Copper Electrodes}

The study of the spatial and electrical characteristics of a bipolar nanosecond discharge in atmospheric pressure air between electrodes from copper showed the following.

At a pulse repetition rate $\mathrm{f}=40-150 \mathrm{~Hz}$, the diameter of the spherical plasma formation in the discharge gap was approximately equal to the interelectrode distance value, and with increasing the repetition rate up to $400-1000 \mathrm{~Hz}$, its diameter increases by 3-4 times, which led to the spread of plasma to new surface areas of the spherical part of the electrodes [19].

The discharge was ignited in an overstressed gap $(d=1$ $\mathrm{mm}$ ) with a weakly nonuniform distribution of the electric field strength between copper electrodes with a spherical working surface with a radius of curvature $3 \mathrm{~mm}$. Its diffuse form and shape, close to spherical, was close to the images of nanosecond discharges in air at interelectrode distances less than $5 \mathrm{~mm}$ with an electrode radius of curvature $6 \mathrm{~mm}$ [14]. In this case, it is considered that runaway electrons can form on working areas of electrodes with natural microprotrusions, which subsequently explode and serve as a source of vapor of electrode material, that is, ectons are formed [15]. The spherical form of plasma formation, which conserved even at high repetition rates, can be associated with the presence of ultraviolet and X-ray radiation, which isotropically highlights the entire discharge gap [16]. As follows from the experimental results [17], in a high-current nanosecond discharge in nitrogen, a beam of runaway electrons is formed only at nitrogen pressures lower than 100 Torr. Therefore, under the conditions of our experiment at an air pressure in the range $p=1-3 \mathrm{Atm}$, the main factor contributing to the 
formation of a diffuse discharge is the ultraviolet and X-ray radiation of the plasma.

By graphically multiplying the oscillograms of the current and voltage pulses, the time distribution of the pulse energy deposition in the plasma of a bipolar nanosecond discharge was obtained. The maximum pulse discharge power was observed in the initial stage of the breakdown of the discharge gap and reached $4 \mathrm{MW}$. Time integration of the pulsed power allowed us to determine the electrical energy that was deposited into the plasma of the discharge under study during a single sequence of voltage and current pulses.
For the conditions of ignition of a bipolar nanosecond discharge at an air pressure $1 \mathrm{Atm}$ and $\mathrm{d}=1 \mathrm{~mm}$, the energy at the maximum reached $100 \mathrm{~mJ}$.

Investigation of spectral characteristics of the radiation of a high-current nanosecond discharge showed that approximately $90 \%$ of its radiation power in the spectral range $200-1000 \mathrm{~nm}$ is concentrated in the spectral range 200$230 \mathrm{~nm}$. In Figure 2 a region of the emission spectrum $(\lambda=$ 200-230 nm) of the discharge plasma in air between copper electrodes is presented. For identification of spectral lines in the spectra of plasma radiation we used directories $[18,19]$.

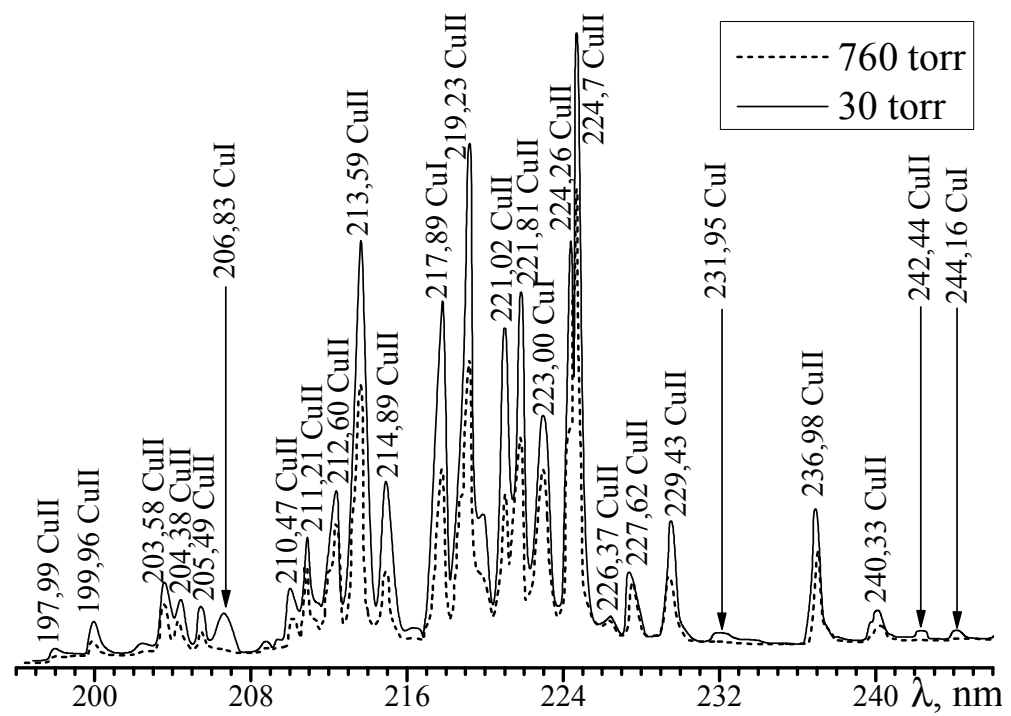

Figure 2. The emission spectrum of a nanosecond discharge plasma in air between copper electrodes.

As for a less powerful unipolar nanosecond discharge between copper electrodes [20], the main lines in the emission spectrum were the spectral lines of copper ions (Table 1). The UV spectrum obtained by us is well correlated with the emission spectrum of a unipolar nanosecond discharge with a beam of runaway electrons and a voltage pulse duration 1-5 ns between copper electrodes (at an interelectrode distance $0.5 \mathrm{~mm}$ ) [21].

Table 1. The results of interpretation the most intense spectral lines in the emission spectrum of an overvoltage nanosecond discharge in air ( $p=1$ Atm) between copper electrodes $(d=1 \mathrm{~mm})$; where: $\lambda$ is the wavelength, $C . S$. - charge state, E - energy state, $K$ - electron configuration, T - term, indices $u^{\prime \prime}$ and $l^{\prime \prime \prime}$ denote upper and lower energy state, I - normalized intensity, taking into account the spectral sensitivity of the photomultiplier and monochromator.

\begin{tabular}{|c|c|c|c|c|c|c|c|c|c|}
\hline № & $\lambda, \mathbf{n m}$ & C. S. & $\mathrm{E}_{\mathrm{l}}, \mathrm{eV}$ & $\mathbf{K}_{\mathbf{l}}$ & $\mathbf{T}_{1}$ & $\mathbf{E}_{\mathbf{u}}$, эB & $\mathbf{K}_{\mathbf{u}}$ & $\mathbf{T}_{\mathbf{u}}$ & I, a. u. \\
\hline 1 & 201,3 & $\mathrm{Cu}$ III & 9,76 & $4 s$ & ${ }^{2} \mathrm{D}_{3 / 2}$ & 15,92 & $4 p$ & ${ }^{2} \mathrm{D}_{3 / 2}^{\mathrm{o}}$ & 56 \\
\hline 2 & 202,4 & $\mathrm{Cu} \mathrm{I}$ & 0,00 & $4 s$ & ${ }^{2} \mathrm{~S}_{1 / 2}$ & 6,12 & $5 p$ & ${ }^{2} \mathrm{P}_{1 / 2}$ & 57 \\
\hline 3 & 202,4 & $\mathrm{Cu} \mathrm{I}$ & 0,00 & $4 s$ & ${ }^{2} \mathrm{~S}_{1 / 2}$ & 6,12 & $5 p$ & ${ }^{2} \mathrm{P}_{3 / 2}$ & 57 \\
\hline 4 & 203,5 & $\mathrm{Cu}$ II & 2,97 & $4 s$ & ${ }^{3} \mathrm{D}_{1}$ & 9,06 & $4 p$ & ${ }^{3} \mathrm{D}_{1}$ & 100 \\
\hline 5 & 204,3 & $\mathrm{Cu}$ II & 2,71 & $4 s$ & ${ }^{3} \mathrm{D}_{3}$ & 8,78 & $4 p$ & ${ }^{3} \mathrm{D}_{3}$ & 52 \\
\hline 6 & 205,4 & $\mathrm{Cu}$ II & 2,83 & $4 s$ & ${ }^{3} \mathrm{D}_{2}$ & 8,86 & $4 p$ & ${ }^{3} \mathrm{D}_{2}$ & 50 \\
\hline 7 & 210,4 & $\mathrm{Cu}$ II & 2,97 & $4 s$ & ${ }^{3} \mathrm{D}_{1}$ & 8,86 & $4 p$ & ${ }^{3} \mathrm{D}_{2}$ & 28 \\
\hline 8 & 211,2 & $\mathrm{Cu}$ II & 3,25 & $4 s$ & ${ }^{1} \mathrm{D}_{2}$ & 9,12 & $4 p$ & ${ }^{1} \mathrm{P}_{1}$ & 47 \\
\hline 9 & 212,6 & $\mathrm{Cu}$ II & 2,83 & $4 s$ & ${ }^{3} \mathrm{D}_{2}$ & 8,66 & $4 p$ & ${ }^{3} \mathrm{~F}_{2}$ & 55 \\
\hline 10 & 213,5 & $\mathrm{Cu}$ II & 2,71 & $4 s$ & ${ }^{3} \mathrm{D}_{3}$ & 8,52 & $4 p$ & ${ }^{3} \mathrm{~F}_{4}$ & 92 \\
\hline 11 & 214,8 & $\mathrm{Cu}$ II & 8,66 & $4 p$ & ${ }^{3} \mathrm{~F}_{2}$ & 14,43 & $4 d$ & ${ }^{3} \mathrm{D}_{2}$ & 32 \\
\hline 12 & 218,1 & $\mathrm{Cu} \mathrm{I}$ & 0,00 & $4 s$ & ${ }^{2} \mathrm{~S}_{1 / 2}$ & 5,68 & $4 s 4 p$ & ${ }^{2} \mathrm{P}_{1 / 2}$ & 43 \\
\hline 13 & 219,2 & $\mathrm{Cu}$ II & 2,83 & $4 s$ & ${ }^{3} \mathrm{D}_{2}$ & 8,48 & $4 p$ & ${ }^{3} \mathrm{~F}_{3}$ & 61 \\
\hline 14 & 219,9 & $\mathrm{Cu} \mathrm{I}$ & 1,38 & $4 s^{2}$ & ${ }^{2} \mathrm{D}_{5 / 2}$ & 7,02 & $4 p$ & ${ }^{2} \mathrm{D}_{5 / 2}$ & 20 \\
\hline 15 & 221,0 & $\mathrm{Cu}$ II & 3,25 & $4 s$ & ${ }^{1} \mathrm{D}_{2}$ & 8,86 & $4 p$ & ${ }^{3} \mathrm{D}_{2}$ & 31 \\
\hline 16 & 221,8 & $\mathrm{Cu}$ II & 2,83 & $4 s$ & ${ }^{3} \mathrm{D}_{2}$ & 8,42 & $4 p$ & ${ }^{3} \mathrm{P}_{1}$ & 39 \\
\hline 17 & 223,0 & $\mathrm{Cu} \mathrm{I}$ & 1,38 & $4 s^{2}$ & ${ }^{2} \mathrm{D}_{5 / 2}$ & 6,94 & $4 s 4 p$ & ${ }^{2} \mathrm{~F}_{7 / 2}$ & 31 \\
\hline 18 & 224,2 & $\mathrm{Cu}$ II & 3,25 & $4 s$ & ${ }^{1} \mathrm{D}_{2}$ & 8,78 & $4 p$ & ${ }^{3} \mathrm{D}_{3}$ & 51 \\
\hline 19 & 224,7 & $\mathrm{Cu}$ II & 2,71 & $4 s$ & ${ }^{3} \mathrm{D}_{3}$ & 8,23 & $4 p$ & ${ }^{3} \mathrm{P}_{2}$ & 61 \\
\hline
\end{tabular}


Table 1 shows the most intense lines in the spectral range $200-250 \mathrm{~nm}$. Spectral lines with intensities greater than $20 \%$ of the most intense in this spectral range are selected. In total, more than 40 spectral lines were recorded. Transitions from the energy states of singly charged ions $8.23-9.12 \mathrm{eV}$ with a change in the electron configuration $4 \mathrm{p}-4 \mathrm{~s}$ dominate in radiation, as well as the spectral lines of atoms arising from transitions from highly excited states with energies 5.68-7.02 $\mathrm{eV}$. When the pressure is reduced to 30 Torr, the integrated radiation intensity of the investigated wavelength range is increased by $30 \%$, and the intensity of individual spectral lines is up to 2 times.

Since under the conditions of the present experiment the main mechanism for the entry into the plasma of copper vapor is ectonic (explosion of micro-points on the surface of the electrodes), in which the electron density of the plasma can reach $10^{16}-10^{17} \mathrm{~cm}^{-3}[20,24]$, the mechanism for the formation of excited copper ions can be determined by processes of excitation of copper ions in the ground state by electrons, as well as electron-ion recombination processes. The effective cross sections of these processes for copper ions have not been investigated at the present time. For zinc ions, the corresponding effective cross sections were obtained and they are sufficiently large $-10^{-16} \mathrm{~cm}^{2}[25,26]$. Therefore, it is possible to assume that excited copper ions are formed in the plasma under investigation as a result of excitation of singly charged copper ions in the ground state by electrons and in the process of dielectronic recombination of doubly charged copper ions with discharge electrons. Within the framework of such a mechanism, an increase in the intensity of the emission of copper ion lines at low air pressure is due to an increase in the electron temperature at high values of the ratio of the electric field strength to the gas density $(\mathrm{E} / \mathrm{N})$. This increases the probability of runaway the electrons from the discharge region. At an air pressure 2-3 Atm, the intensity of spectral lines of copper ions is about 1.5-2 times higher than for $\mathrm{p}=1 \mathrm{Atm}$. This may be due to an increase in the efficiency of recombination processes at high air pressure.

Estimated calculations of some plasma parameters are carried out for a low average density of copper vapor in the discharge gap $(\mathrm{p} \mathrm{Cu})=30$ Па). Small partial pressures in discharge gap of the copper vapor in the present experiment are indicated by small pulse repetition rates $(100-1000 \mathrm{~Hz})$ and small autographs on the working surface of the electrodes. As in [24], traces of erosion were the appearance of individual points with a diameter of up to 100-200 microns, which relatively evenly filled the working surface of the electrodes.

In the case of strong electron runaway, the standard procedure for finding the electron energy distribution function (EEDF) by means of a method that is being realized with a help of programs such as BOLSIG + or the program [25] is incorrect and more complex calculations are required [26]. Under the conditions of this experiment, the maximum value of the parameter E/P reaches (at air pressure $\mathrm{p}=1 \mathrm{Atm}$ and $\mathrm{d}=1 \mathrm{~mm}$ ) approximately $530 \mathrm{~V} \mathrm{~cm}^{-1} \mathrm{Torr}^{-1}$, which is less than the critical value $\mathrm{E} / \mathrm{P}$ for nitrogen according to the local electron runaway criterion $-590 \mathrm{~V} \mathrm{~cm}^{-1} \mathrm{Torr}^{-1}$ [27]. A small number of runaway electrons can appear due to the amplification of the electric field on the natural micro-points located on the working surfaces of the electrodes. On the kinetics of processes in the plasma, they affect little, since the effective cross sections of all electron-atom and electronmolecular processes decrease significantly at high electron energy. Therefore, for estimating the plasma parameters of atmospheric pressure, we chose the standard program for solving the Boltzmann kinetic equation for EEDF.

The parameters of the discharge plasma in air mixture with a small addition of copper vapor at atmospheric pressure (component ratio $101 \mathrm{kPa}: 30 \mathrm{~Pa}$, respectively) were determined numerically and calculated as complete integrals of the EEDF in the discharge. The EEDF were numerically determined by solving the Boltzmann kinetic equation in two-term approximation. The EEDF calculations were carried out using the program [25]. On the basis of the EEDF obtained, mean electron energy, the electron mobility are determined as a function of the magnitude of the reduced electric field (the ratio of the electric field strength (E) to the total concentration of air atoms and molecules consisting of a natural mixture of argon, molecules of carbon dioxide, oxygen and nitrogen, and a small admixture of copper vapor $(\mathrm{N})$ ). The range of the parameter change $\mathrm{E} / \mathrm{N}=1-1300 \mathrm{Td}$ $\left(1 \cdot 10^{-17}-1 \cdot 10^{-15} \mathrm{~V} \mathrm{~cm}^{2}\right)$ included the values of the parameter $\mathrm{E} / \mathrm{N}$, which were realized in the experiment. In the integral of electron collisions with atoms and molecules, the following processes are taken into account: elastic scattering of electrons by copper atoms, excitation of energy states of copper atoms (threshold energy $1.500 \mathrm{eV}, 3.800 \mathrm{eV}, 5.100$ $\mathrm{eV}$ ), ionization of copper atoms (threshold energy $7.724 \mathrm{eV}$ ); elastic scattering of electrons by copper atoms, excitation of the energy state of argon atoms (threshold energy $11.50 \mathrm{eV}$ ), ionization of argon atoms (threshold energy, $15.80 \mathrm{eV}$ ); elastic scattering and excitation of the energy states of carbon dioxide molecules: vibrational (threshold energies: $0.083 \mathrm{eV}$, $0.167 \mathrm{eV}, 0.252 \mathrm{eV}, 0.291 \mathrm{eV}, 0.339 \mathrm{eV}, 0.422 \mathrm{eV}, 0.505 \mathrm{eV}$, $2.5 \mathrm{eV}$ ), electronic (threshold energies: $7.0 \mathrm{eV}, 10.5 \mathrm{eV}$ ), dissociative attachment of an electron (threshold energy $3.85 \mathrm{eV}$ ), ionization (threshold energy $13.30 \mathrm{eV}$ ); elastic scattering and excitation of energy states of oxygen molecules: vibrational (threshold energy: $0.190 \mathrm{eV}, 0.380 \mathrm{eV}$, $0.570 \mathrm{eV}, 0.750 \mathrm{eV}$ ), electronic (threshold energy: $0.977 \mathrm{eV}$, $1.627 \mathrm{eV}, 4.500 \mathrm{eV}, 6.000 \mathrm{eV}, 8.400 \mathrm{eV}, 9.970 \mathrm{eV}$, dissociative attachment of an electron (threshold energy $4.40 \mathrm{eV}$ ), ionization (threshold energy - $12.06 \mathrm{eV}$ ); elastic scattering and excitation of energy states of nitrogen molecules: rotational - threshold energy $0.020 \mathrm{eV}$, vibrational (threshold energy: $0.290 \mathrm{eV}, 0.291 \mathrm{eV}, 0.590 \mathrm{eV}, 0.8801 .170$, 1.470, 1.760, 2.060, 2.350, electronic: $6.170 \mathrm{eV}, 7.000$, $7.350,7.360,7.800,8.160,8.400,8.550,8.890,11.03,11.87$, $12.25,13.00$, ionization (threshold energy is $15.60 \mathrm{eV}$ ) The data on the absolute values of the effective cross sections of 
these processes, as well as their dependences on the electron energies, are taken from the database [25].

In Figure 3 the dependences of mean electron energy and the mobility of electrons are presented as a function of the reduced electric field. Mean energy of the discharge electrons depends most strongly on the parameter $\mathrm{E} / \mathrm{N}=1-200 \mathrm{Td}$, while it increases linearly from 0.15 to $8.77 \mathrm{eV}$. In the range of the parameter $\mathrm{E} / \mathrm{N}=400-1300 \mathrm{Td}$, mean electron energy also increases from 8.77 to $29.26 \mathrm{eV}$, but at a slower rate. For the range of reduced electric field strength $615 \mathrm{Td}-820 \mathrm{Td}$ at which experimental electrical and optical characteristics of the discharge (the voltage amplitude was within 15-20 kV) were carried out, mean electron energies varied within the limits $12.6-16.5 \mathrm{eV}$. And their largest energies corresponded to values $177-300 \mathrm{eV}$.

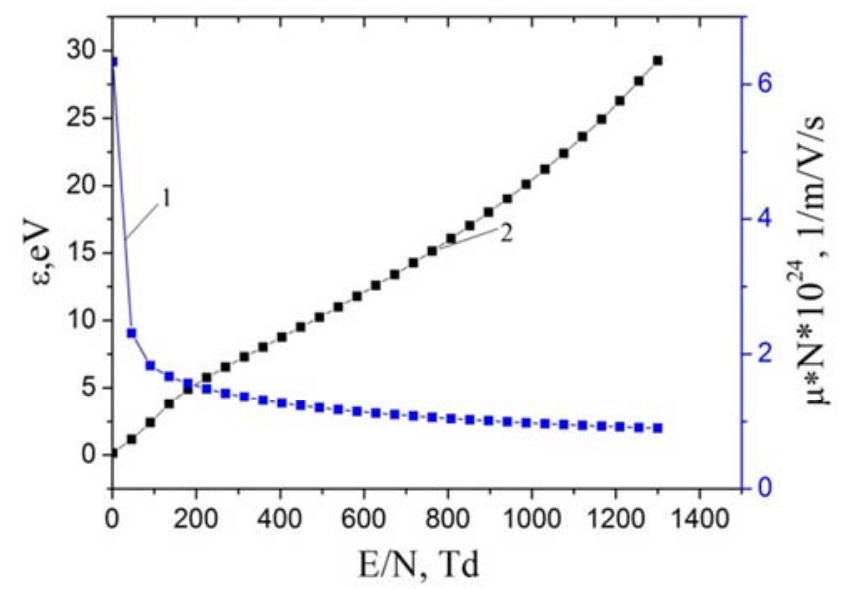

Figure 3. The dependence of the product of electron mobility on the total concentration of the components of the mixture of copper vapor and air (curve 1) and their mean energy (curve 2) on the reduced electric field strength.
The product of the electron mobility to the total concentration of the components of the mixture of copper vapor and air $\left(\mathrm{N}=2.44 \cdot 10^{25} \mathrm{~m}^{3}\right)$, as follows from the numerical calculation (Figure 3), varies within the range $1.113010^{24} \cdot \mathrm{N}-1.031 \cdot 10^{24} \cdot \mathrm{N}(1 / \mathrm{m} / \mathrm{V} / \mathrm{s})$ with a change in the parameter $\mathrm{E} / \mathrm{N}$ in the range $615 \mathrm{Td}-820 \mathrm{Td}$, which gives the values of the electron drift velocity $6.8 \cdot 10^{4} \mathrm{~m} / \mathrm{s}$ and $8.4 \cdot 10^{4} \mathrm{~m} / \mathrm{s}$, respectively, for the field strength on the plasma $15.0 \cdot 10^{6} \mathrm{~V} / \mathrm{m}$ and $20.0 \cdot 10^{6} \mathrm{~V} / \mathrm{m}$. The electron concentrations for the reduced electric field strength $615 \mathrm{Td}$ to $820 \mathrm{Td}$ had values $70.3 \cdot 10^{21} \mathrm{~m}-3-56.9 \cdot 10^{21} \mathrm{~m}^{-3}$ at a current density $765 \cdot 10^{6} \mathrm{~A} / \mathrm{m}^{2}$ on the surface of copper electrodes $\left(0.196 \cdot 10^{-6}\right.$ $\mathrm{m}^{2}$ ).

\subsection{Optical Characteristics of thin Nanostructured Films}

Experiments on the synthesis of thin nanostructured films were carried out from the products of sputtering copper electrodes at an air pressure $1 \mathrm{Atm}$ and an interelectrode distance $1 \mathrm{~mm}$. In Figure 4 an image of the surface of the film deposited on the surface of the glass substrate is given. To determine the size of nanostructures, a gel based on standard spherical nanostructures of gold with a diameter 20 $\mathrm{nm}$ was applied to the surface of the film (it is highlighted in yellow in the image). A comparative analysis of the sizes of spherical gold nanostructures and nanostructures synthesized in the reactor showed that the sizes of synthesized nanostructures are in the range $2-20 \mathrm{~nm}$. The transverse section of the nanostructures (in the direction perpendicular to the substrate, has not been studied, but it can be assumed that these are nanowhiskers of copper oxides.) When the energy input to the plasma is increased (magnetrons or liquid discharges), nanowhiskers synthesis of similar nanostructures is usually fixed [28].

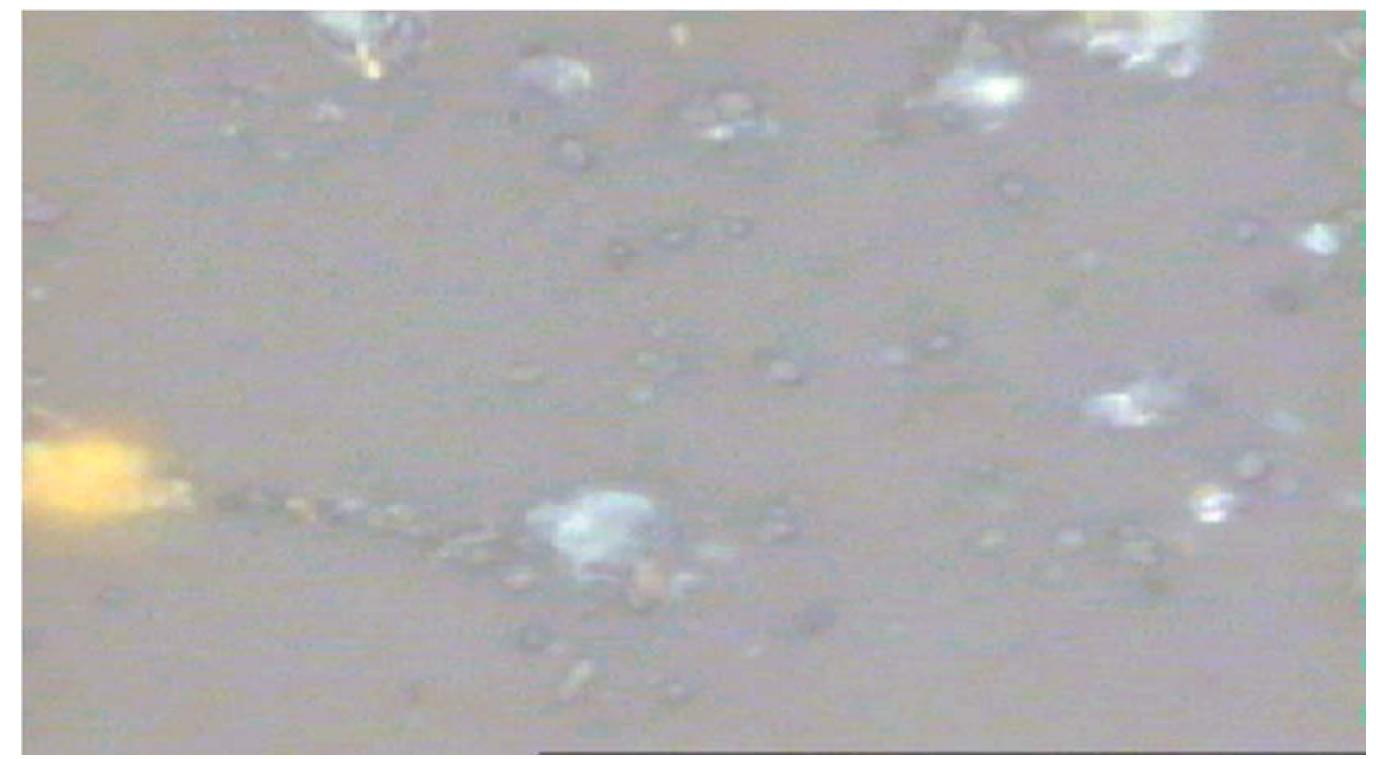

Figure 4. The surface structure of a thin film synthesized on a glass substrate from products of sputtering copper electrodes in atmospheric pressure air for 30 minutes under the action of a bipolar nanosecond discharge $(d=1 \mathrm{~mm}$ and $f=100 \mathrm{~Hz})$.

The transmission spectrum of the film in the visible region is shown in Figure 5 [29]. 


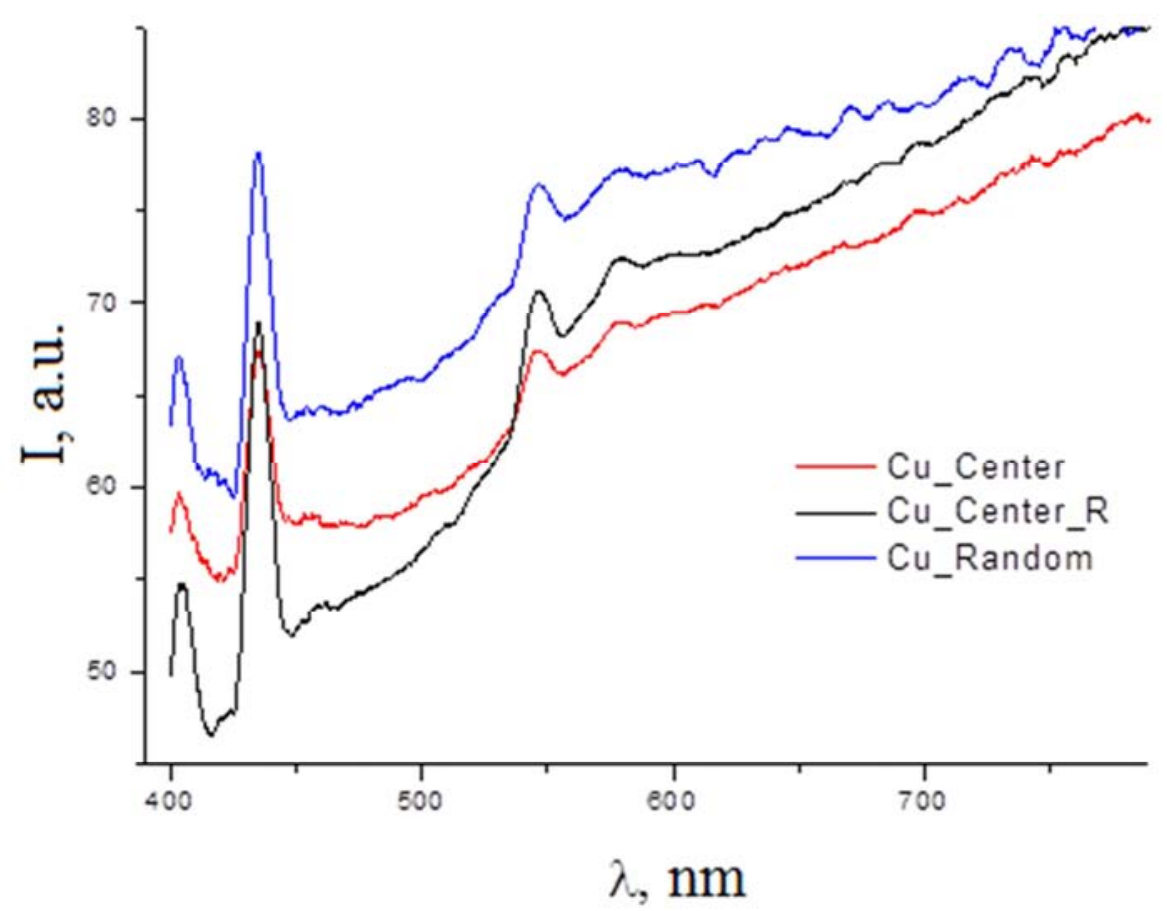

Figure 5. Transmission spectra of thin films synthesized by sputtering copper electrodes in overvoltage bipolar nanosecond discharge, obtained for different regions of the substrate: the center of the substrate (red color), the middle of the substrate (black color) and the edge of the substrate (blue color).

The nonuniformity of the film along the radius of the substrate is due to the use of a point source of vapor of the material of the electrodes and can be overcome when the substrate moves relative to the discharge at a certain slow speed. The general form of the bandwidth of nanostructures, when the transmission of the film increased sharply in the visible wavelength range (continuum from 500 to $650 \mathrm{~nm}$ ), well corresponds to the transmission of copper oxide nanostructures $\left(\mathrm{Cu}_{2} \mathrm{O}\right)$ [30]. The absorption band of the film in the wavelength range $550-580 \mathrm{~nm}$ correlates well with the absorption band of copper nanostructures [4]. Narrow bands in the blue region of the spectrum (at wavelengths 420 and $450 \mathrm{~nm}$, are most likely associated with the action of ultraviolet radiation in the spectral range of $200-230 \mathrm{~nm}$ on film, which can lead to radiation defects in nanostructures based on oxide and copper protoxide and formation new energy states in the band gap of these compounds. For nanostructures of copper oxide and copper protoxide and their colloidal solutions are characteristic the broad structureless transmission spectra in the ultraviolet, visible and near infrared regions of wavelengths [31, 2], but not so narrow enlightened sections of which have been observed in our experiment.

Photoluminescence was most efficiently excited from radiation with a wavelength $330 \mathrm{~nm}$. The photoluminescence spectra were characterized by two broad maxima in the spectral range 400-600 $\mathrm{nm}$, which shifted to the longwavelength region of the spectrum and decreased in intensity when passing from the wavelength of the exciting radiation at $330 \mathrm{~nm}$ both to the $270 \mathrm{~nm}$ region as well as to the $380 \mathrm{~nm}$ region [33, 34]. In [35] it is pointed out that assisting the growth of transparent conducting layers based on zinc oxide with ultraviolet radiation from a mercury lamp improves their electrical characteristics by creating additional donor centers and reducing the scattering of charge carriers at the grain boundaries of nanowhiskers. In our case, there is no need to use an external source of ultraviolet radiation, since the plasma itself is a selective radiator in the spectral range 200$230 \mathrm{~nm}$.

\section{Conclusions}

It is shown that the overstressed nanosecond discharge in atmospheric pressure air between electrodes from copper is a selective source of ultraviolet radiation of copper ions in the spectral range $200-230 \mathrm{~nm}$; the maximum pulsed energy deposition in the plasma is $4 \mathrm{MW}$, and the energy deposited in the discharge per pulse is approximately $0.1 \mathrm{~J}$; the most probable mechanism for excitation of UV radiation from copper ions is the ectonic mechanism of erosion of the surface of copper electrodes, the transfer of energy to copper atoms from nitrogen molecules in the metastable state, the further excitation and ionization of copper atoms and ions by electron impact, and the formation of $\mathrm{Cu}^{+*}$ ions in electronion recombination of doubly charged copper ions and electrons. Estimates of the plasma parameters on the basis of the solution of the Boltzmann equation for the electron energy distribution function have shown that for the range of the reduced electric field strength $(615-820) \mathrm{Td}$ at which the experiments were performed, mean electron energy varied in the range $12.6-16.5 \mathrm{eV}$. The electron concentration is in the range $(57-70) \cdot 10^{21} \mathrm{~m}^{-3}$.

Thin nanostructured films based on copper nanostructures and copper oxides have been synthesized, under the influence of ultraviolet assisting by plasma radiation of an overstressed nanosecond discharge emitting in the spectral range $200-230 \mathrm{~nm}$. 


\section{References}

[1] Apollonov, V. V. and N. V. Pletnev N. V. (2012). Kvantovaya Elektronika 20, 6, 670-674.

[2] Abaeva, L. F., Shumskiy, V. I., Petritskaya, E. N., Rogatkin, D. A., P. N. Lyubchenko (2010). Al'manakh klinicheskoy meditsiny 22, 10-16. (in Russian).

[3] Bobkov, A. A., Lashkova, N. A., Maximov, A. I., Moshnikov, V. A. and S. S. Nalimova (2017). Fizika i Tekhnika Poluprovodnikov 51, 1, 63-67.

[4] Farmanfarmaei, B., RashidianVaziri, M. R. and F. Hajiesmaeilbaigi (2014). Kvantovaya Elektronika 44, 11, 1029-1032.

[5] Tyurnina, A. E., Shur, V. Ya., Kozin, R. V., Kuznetsov, D. K., Pryakhina, V. and G. V. Burban (2014). Physics of the Solid State $56,7,1431-1437$.

[6] V. S. Burakov, V. S., Kiris, V. V., Nevar, A. A., Nedelko, M. I. and N. V. Tarasenko (2016) Journal of Applied Spectroscopy $83,4,643-649$.

[7] Rutberg, F. G., Gusarov, V. V., Kolikov, V. A., Voskresenskaya, I. P., Snegov, V. N., Stogov, A. Yu. and I. A. Cherepkova (2012) Technical Physics 57, 12, 1641.

[8] Babich, L. P. (2003) Futurepast. Arlington. Virginia. ITS Science and Technology Series, 2, 353.

[9] V. F. Tarasenko, Runaway electrons preionized diffuse discharge. New York: Nova Science Publishers Inc., 2014, 578 .

[10] Erofeev, M. V., Shulepov, M. A. and V. F. Tarasenko (2016) International Journal of Applied and Fundamental Research 1, 8-13.

[11] Shuaibov, A. K., Minya, A. I., Enedi, A. L., Shevera, I. V., Gomok, Z. T. and V. V. Danilo ( 2017) Elektronnaya obrabotka materialov 53, 2, 45-49.

[12] Shevera, I. V, Minya, A . I., Shuaibov, OK., Danilo, V. . and Z. T. Homoki (2017) Scientific herald of Uzhgorod University. Physics 41, 113-120.

[13] Shuaibov, Alexsander, Mynia, Oleksandr, Chuchman, Mykhaylo, Homoki, Zoltan and Vladislav Danylo (2017) Characteristics of overstrained nanosecond discharge in a mixture of air wich copper steam. Proceedings of the XIII International Conference "Electronics and Applied Physics", Kyiv, Ukraine, Taras Shevchenko National University, 151152.

[14] Babich, L. P., Loiko, T. V. and V. A. Tsukerman (1990) Physics-Uspekhi 33, 7, 521-540.

[15] Mesyats, G. A. (1995) Physics-Uspekhi 165, 6, 601-626.

[16] Bakst, T. Kh., Tarasenko, V. F., Shut ko, Yu. V. and M. V. Erofeev (2012) Qantum Electronocs 42, 2, 153-156.

[17] Baksht, E. Kh., Burachenko, A. G., Lomaev, M. I., Panchenko, A. N. and V. F. Tarasenko (2015) Kvantovaya Elektronika 45, 4, 366-370.
[18] Smith, P. L., Heise, C., Esmond, J. R. and R. L. Kurucz, Atomic Spectral Line Database from CD-ROM 23 of R. L. Kurucz (Smithosian Astrophysical Observatory, Cambridge, 1995. http://cfa-www.harvard.edu/amp).

[19] Sansonetti, J. E. and W. C. Martin (2005) J. Phys. Chem. 34, 1559.

[20] Shuaibov, A. K. Laslov, G. E. and Ya. Ya. Kozak (2014) Optics and Spectroscopy 116, 4, 552-556.

[21] Avtaeva, S. V., Zhdanova, O. S., Pikulev, A. A., Sosnin, E. A. and V. F. Tarasenko, New Direction in Scientific Research and Application of Excilams, Tomsk. STT. Publishing, 2013, 246.

[22] Levko, Dmitry and Laxminarayan L. Raja (2016) Physics of Plasmas, 22, 123518.

[23] A. N. Gomonai (2015) Journal of Applied Spectroscopy 82, 1, 17-22.

[24] Tren'kin, A. A., Karelin, V. I., Shibitov, Yu. M., Blinova, O. M. and I. S. Yasnikov (2017) Technical Physics 87, 9, 1419.

[25] http:/www.bolsig.laplace.univ-tlse.fr.

[26] Dmitry Levko (2013) Journal of Applied Physics 114, 223302.

[27] Tarasenko, V. F., and, S. I. Yakovlenko (2004) PhysicsUspekhi 47, 887-905.

[28] Bulychev, N. A., Kazaryan, M. A., Nikiforov, V. N., Shevchenko, S. N., Yakunin, V. G., Timoshenko, V. Yu, Bychenko, A. B. and V. G. Sredin (2016) Technical Physics Letters 42, 5, 495-497.

[29] Shevera, I. V., Minya, O. I., Shuiabov, OK., Sichka, M. Yu. Danilo, V. V. and Z. T. Gomoki (2017) Spectra of transmission (absorption) of thin nanostructured films based on $\mathrm{Zn}, \mathrm{Cu}, \mathrm{Fe}$ and their oxides. Proceedings of the international conference of young scientists and post-graduate students, Uzhhorod, $152-153$.

[30] Zamanova, L. A. and L. A. Alieva (2008) Fizika. Cild XIV, 3, 194-196.

[31] Mynia, Oleksandr, Shuaibov, Alexsander, Holomb, Roman, Danylo, Vladislav, Shevera, Ighor, and Volodymyr Mitsa (2017) Synthesis of Nanostructures under Action of the strongly overstrained nanosecond Discharge in the Air atmospheric pressure. Proceedings of the XIII International Conference "Electronics and Applied Physics", Kyiv, Ukraine, Taras Shevchenko National University, 153-154.

[32] Kudryashov, D. A., Gudovskih, A. S., Babichev, A. V., Filimonov, A. V., Mozharov, A. M., Agekyan, V. F., Borisov, E. V., Serov, A. Yu. and N. G. Filosofov (2017) Semiconductors 51, 1, 110-114.

[33] O. A. Gololobova (2015)Izvestiya of the Samara Scientific Center of the Russian Academy of Sciences 17, 2, 69-74.

[34] Shuaibov, A. K., Minya, A. I., Gomoki, Z. T. and V. V. Danilo, Method of nanostructuring of glass surface in atmospheric pressure air// Patent (utility model) №. 120612, November 10, 2017 Bulletin number №. 21, Uzhgorod National University.

[35] Abduev, A. Kh., Asvarov, A. Sh., Akhmedov, A. K., Emirov, R. M. and V. V. Belyaev (2017) JETF letters, 43, 22, 40-47. 